\title{
Interaction of Interstellar Shocks with Dense Obstacles: Formation of "Bullets"
}

\section{V.V. Gvaramadze}

Abastumani Astrophysical Observatory, Tbilisi, Georgia

\begin{abstract}
The so-called cumulative effect take place in converging conical shock waves arising behind dense obstacles overtaken by incident interstellar shock. A significant part of energy of converging flow of matter swept-up by a radiative conical shock can be transferred to a dense jet-like ejection ("bullet") directed along the cone axis. Possible applications of this effect for star-forming regions (e.g., OMC-1) and supernova remnants (e.g., Vela SNR) are discussed.
\end{abstract}

\section{Introduction}

Recently in some galactic objects were discovered unusual linear structures radially diverging from the common origin. The most striking examples of this phenomenon were observed in the Orion molecular cloud (Allen \& Burton 1993) and the Vela supernova remnant (Aschenbach et al. 1995). These structures look like wakes trailed behind dense "bullets" moving with supersonic velocities through the ambient medium. The fact that wakes have approximately equal projected extents suggests the simultaneous origin of "bullets", possible, in an explosive event. Allen \& Burton (1993) and Aschenbach et al. (1995) suggested that the "bullets" were ejected directly from the central source of activity (young massive star and supernova star respectively). We propose (Gvaramadze 1997a,b,c) an alternative explanation based on the interaction of a spherical shock wave with dense spherical obstacles (e.g., cloudlets or stellar wind zones). The "bullets" result from cooling and condensation of a gas swept-up by converging conical shocks arising behind obstacles overtaken by the incident (spherical) shock (see Fig. 1). The role of obstacles consists only in a transient focusing of the incident shock.

\section{Formation of "Bullets" in Radiative Converging Conical Shock Waves}

A converging conical shock forms (see Fig. 1) when a strong shock overtakes a spherical obstacle. The height of the cone is approximately equal to the radius of the obstacle. The cone half-opening angle $\nu$ depends on the curvature of the incident shock; for plane shocks, $\nu \simeq 35^{\circ}$ (e.g., Tenorio-Tagle \& Rozyczka 1984). 


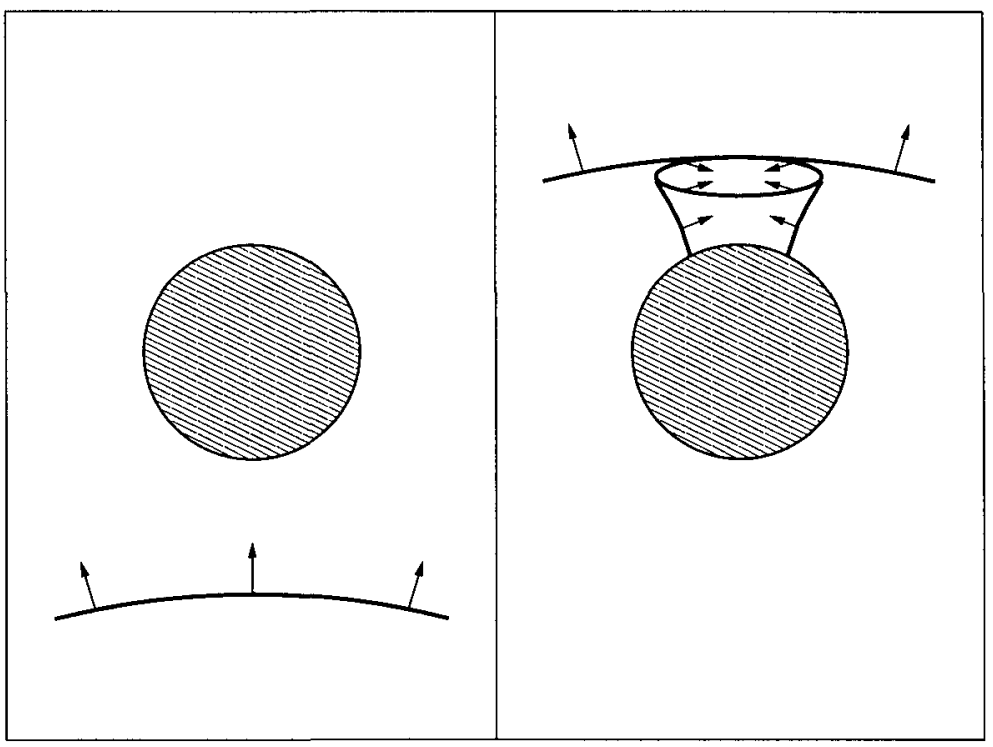

Fig. 1. Formation of a converging conical shock behind a dense obstacle.

An important property of a conical shock wave is that it creates, due to the Mach reflection, an axial pressure gradient which drives a high-velocity mass ejection along the cone axis (for details and references see Gvaramadze 1997a,b). Effects of such type are called cumulative (see Gvaramadze (1996) and references therein). The velocity of ejection (the so-called cumulative jet) is given by, $v_{\mathrm{ej}} \simeq U / \sin \nu$, where $U$ is the velocity of the converging conical shock, $\nu$ is the cone half-opening angle; $v_{\mathrm{ej}} \simeq 1.7 \mathrm{U}$ for $\nu=35^{\circ}$. It is obvious that due to the finite dimensions of real conical flows, the formation of cumulative ejections is a transient process. Furthermore, the ejection in itself is unstable because the overpressure, created by the converging flow, tends to re-expand it. A significant step in understanding of formation of ejections in converging conical shocks was made by Tenorio-Tagle \& Rozyczka (1984). They shown that the radiative cooling of shock waves favours the formation of long-living ejections ("bullets"). The sound speed inside a dense and cold "bullet" formed in a radiative conical shock can be much smaller than the velocity of directed motion. Such a "bullet" can propagate through the ambient medium without significant dispersion.

\section{Formation of the Fan of Ejections in the OMC-1}

The reader is referred to Gvaramadze (1997a,b), who describes the formation of the fan of ejections in the OMC-1 in detail. 


\section{Origin of X-ray Protrusions in the Vela SNR}

The optical observations (e.g., Parker et al. 1979) and the X-ray observations (e.g., Aschenbach et al. 1995) of the Vela SNR reveal very complex and asymmetrical structure of this remnant (see Fig. 2), with some features extended up to $\simeq 5^{\circ}$ from the center (associated with the Vela pulsar position). We suggest (Gvaramadze 1997c) that the shape of the Vela SNR might be explained as the result of interaction of the SN ejecta/shock with the preexisting wind-driven shell (WDS) created in the interstellar medium with a density gradient perpendicular to the Galactic plane. The mass of the WDS is a decisive factor, which determines the evolution of the SN shock. If the mass of the WDS is smaller than $\simeq 50 M_{\odot}$, the SN shock overruns the shell and continues to evolve adiabatically (e.g., Franco et al. 1991). For more massive shells, the SN shock losses, due to the radiative cooling, a significant part of their energy and merges with the WDS. In this case, the SN remnant evolves as a radiative shock. These two cases can coexist in one and the same SN remnant if the WDS was blown-up in the density stratified medium. Apparently just this situation take place in the case of Vela SNR.

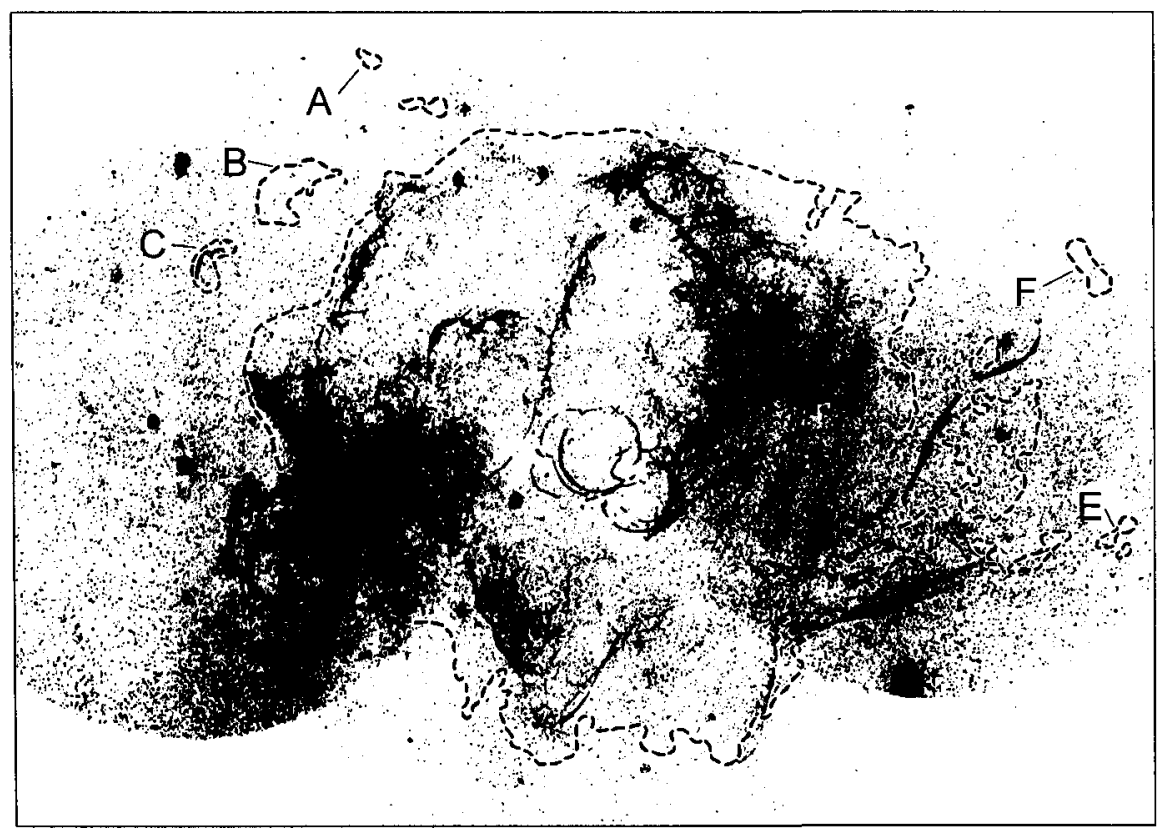

Fig. 2. Mosaic of [O III] $\lambda 5010$ filter photographs of the Vela SNR (Parker et al. 1979). The boundary of the ROSAT image of the remnant (Aschenbach et al. 1995) is outlined by dashed contours. Position of the Vela pulsar is indicated by a cross. 
The number density of the interstellar medium, $n_{0}$, in the direction towards the Galactic plane can be estimated from the consideration of the dynamics of the "bullet" A (see Strom et al. 1995). It is connected with the number density inside the bullet, $n_{\mathrm{b}}$, through the simple relation: $n_{0} \simeq n_{\mathrm{b}} r_{\mathrm{b}} / L$, where $r_{\mathrm{b}}$ is the characteristic size of the "bullet" ( $\left.\simeq 1 \mathrm{pc}\right), L$ is the distance it has travelled. Provided the "bullet" was ejected from the SN explosion site, i.e. $L \simeq 50 \mathrm{pc}$, and for $n_{\mathrm{b}} \simeq 0.4-0.9 \mathrm{~cm}^{-3}$ (Strom et al. 1995, Aschenbach et al. 1995), it follows from the above relation that $n_{0} \simeq 0.01-0.02 \mathrm{~cm}^{-3}$. However, $n_{0}$ is few times higher than it was estimated by Strom et al. (1995) if the "bullet" was formed at some distance $(\geq 30 \mathrm{pc})$ from the center. We adopt the value $n_{0} \simeq 0.03 \mathrm{~cm}^{-3}$. In this case, the mass of the WDS of radius $\simeq 30 \mathrm{pc}$ is $\geq 50 M_{\odot}$. This means that the SN shock cannot overrun the norteast half of the WDS faced towards the local density increase (see, e.g., Dame et al. 1987) and evolves as a radiative one. The interaction of this (radiative) half of the SN shock with dense obstacles (cloudlets, stellar wind zones) can produce cumulative ejections observed by Aschenbach et al. (1995) as X-ray "bullets" A, B and C. Note that the X-ray "bullet" C has an optical counterpart (see Fig. 2), consisting of two arc-like filaments resembling bow shocks.

The impact of the SN shock with the southwest (less massive) half of the WDS leads to its strong deformation and even disruption in places (see Fig. 2). A hot gas of SN interior escapes through the gaps on the SN shell and forms two outflows partially bounded by filamentary structures (see Fig. 2). These outflows appear as the X-ray protrusions $\mathrm{E}$ and $\mathrm{F}$ (Aschenbach et al. 1995).

Acknowledgements. The work was supported by the Deutscher Akademischer Austauschdienst (DAAD) and the Deutsche Forschungsgemeinschaft (DFG).

\section{References}

Allen D.A., Burton M.G. (1993): Nat 363, 54

Aschenbach B., Egger R., Trümper J. (1995): Nat 373, 587

Dame T.M. et al. (1987): ApJ 322, 706

Franco J., Tenorio-Tagle G., Bodenheimer P., Rozyczka M. (1991): PASP 103, 803 Gvaramadze V.V. (1996): In W.Kundt (ed.), Jets from Stars and Galactic Nuclei, Springer-Verlag, Berlin, p. 92

Gvaramadze V.V. (1997a): In F.Malbet, A.Castets (eds.), Low Mass Star Formation

- from Infall to Outflow, poster proceed. of IAU Symp. No. 182, p. 129

Gvaramadze V.V. (1997b): Astronomy Letters 23, No. 8, in press

Gvaramadze V.V. (1997c): in preparation

Parker A.R., Gull T.R., Kirschner R.P. (1979): An Emission-Line Survey of the Milky Way, NASA SP-434

Strom R., Johnston H.M., Verbunt F., Aschenbach B. (1995): Nat 373, 590

Tenorio-Tagle G., Rozyczka M. (1984): A\&A 141, 351 